\title{
TECNOLOGÍA AL SERVICIO DE LA EMPRESA Y LA SOCIEDAD*
}

\author{
Carmen Rosa Graham \\ carmen.graham@gmail.com \\ Presidenta del Consejo Consultivo de la \\ Facultad de Ingeniería de Sistemas \\ Universidad de Lima
}

\section{Resumen}

En las últimas dos décadas hemos participado de cambios fundamentales en el concepto empresarial, desde la organización que "trabaja hacia dentro" en busca de mayor productividad, hasta la empresa que lidera o forma parte de un ecosistema empresarial que, trabajando en ambiente colaborativo, desarrolla sinergias para lograr competitividad.

Nada de esto sería posible sin la mentalidad innovadora de los ejecutivos y el uso intensivo de la tecnología de la información y de las comunicaciones. Empresa exitosa y tecnología hoy son un binomio, y la oferta tecnológica cada vez esta más cercana en cuanto a soluciones pragmáticas y alternativas financieras.

La incorporación de la tecnología a la vida diaria de las empresas y personas, trae importantes oportunidades en educación y salud, como ningún otro momento en la historia. Hoy la disponibilidad de la tecnología brinda mayor acceso incluso a aqueIlas comunidades que están geográficamente aisladas. Es pues sin duda la gran oportunidad de desarrollar conocimiento y, con ello, una sociedad mejor.

Palabras clave: Tecnologías de la información y empresa Ecosistema empresarial, Ambiente colaborativo e innovación, Evolución empresarial y tecnológica. 


\section{Introducción}

Para comentar sobre tecnología en estos días, es imprescindible hablar sobre cambio e innovación, en nuestra vida cotidiana y en la empresa. Innovación con la que vamos alcanzando distintos modelos tanto empresariales como sociales. Así, en este documento nos referiremos a los modelos empresariales y a la sociedad del conocimiento como dos importantes elementos del mundo de hoy y de los próximos años.

Atravesamos por un momento interesantísimo en el que la tecnología, que vimos desarrollarse por años y que parecía ciencia ficción por lo compleja, aparatosa y lejana, hoy comienza a hacer un perfecto maridaje con los negocios y la sociedad. Situación especialmente importante porque al involucrarse mayor cantidad de usuarios e interesados, los desarrollos de nuevas tecnologías tienden a hacerse más prácticos, fáciles de usar y simples. Esto nos da la seguridad de que en el futuro próximo veremos aún mejores resultados.

Refirámonos en principio a la empresa, y para ello quisiera citar el "Global CEO Study 2006" (7) que desarrolla IBM con 765 personas influyentes de los sectores empresarial y público en el ámbito mundial. En este estudio el 65 por ciento de los entrevistados dice que tiene que hacer cambios fundamentales en sus empresas e instituciones. Sigue siendo muy importante la innovación en productos y servicios, pero le dan especial énfasis a diferenciarse en el mercado, innovando desde lo más elemental sus modelos de negocio.

\section{Evolución en el enfoque empresarial}

Vemos así una notable evolución en solo unos pocos años, Primero fue el foco de la gerencia en desarrollar y comercializar un producto o servicio nuevo, que podía ser imitado o copiado por otra empresa rápidamente y la novedad del producto no era ya tal. Esto hoy es solo una actividad más que desarrolla la empresa pero no es la prioritaria.

En una segunda etapa pasamos a la optimización de costos (léase reducción en la mayoría de los casos) y de procesos operativos como temas prioritarios, los cuales fueron un imperativo de rentabilidad y subsistencia pero no daban una ventaja competitiva de largo plazo, porque también las técnicas y métodos eran perfectamente replicables.

Y hoy, cuando las empresas líderes ya van logrando ventajas competitivas en producto/servicio y en operaciones, así como procesos para mantener ambos renovándose continuamente, están enfocadas en defi- 
nir un nuevo modelo de negocios y en construir alianzas estratégicas que les permitan crear sinergias o complementar sus procesos no esenciales (core). Este nuevo modelo no es fácil de replicar porque construirlo toma tiempo y mucho esfuerzo, y no parecen existir dos empresas con la misma estrategia y, sobre todo, con las mismas posibilidades.

Esta es una etapa aún más compleja que las anteriores. En aquellas oportunidades la mayor parte del éxito estaba en la correcta aplicación de técnicas y metodologías. La experiencia exitosa de una empresa podía ser replicada a satisfacción en otra empresa, y si bien los cambios causaban impacto en la organización, era todo bastante más estructurado y, además, existen metodologías para tratarlo. Conocimientos específicos eran adoptados o desarrollados por la empresa y el éxito o fracaso dependía de una correcta implementación, que por lo general se hacía con "expertos internos" y un consultor externo.

En la etapa actual, con el incremento del comercio internacional y la velocidad que proporciona la tecnología, la empresa tiene que ser competitiva y exitosa, al mismo tiempo que se reinventa. Vemos casos en que la empresa sabe que la línea de productos que hoy proporciona más del 50 por ciento de sus ventas está por colapsar por un cambio tecnológico y de preferencia de sus clientes, o que el servicio que venía prestando con una fuerza de ventas especializada, hoy ha sido reemplazada por un call center. Esto es perfectamente válido tanto para grandes empresas como Kodak y Sony, como para cualquier pequeña empresa de nuestro entorno.

\section{Ecosistema empresarial}

Otra característica de la etapa de innovación actual es que el éxito no depende solamente del esfuerzo que hagan los inversionistas y ejecutivos para innovar "su" estructura organizacional interna, sino de la habilidad que tengan para construir, pertenecer o liderar un ecosistema empresarial y hacer que este sea exitoso.

El referirnos a un ecosistema empresarial es mucho más preciso que el concepto de cadena de suministro, pues el ecosistema es más un conglomerado que funciona ordenadamente, a un solo ritmo, y donde los agentes o elementos son algunos internos y la mayoría externos a la empresa. Es así que el éxito de la empresa depende no sólo del conocimiento y las acciones internas sino de cómo trabaja el conjunto de agentes.

Al ecosistema pueden pertenecer empresas de diversas dimensiones y ubicaciones, aliados estratégicos, grupos de influenciadores (trabaja- 
dores, clientes, etcétera), instancias gubernamentales y académicas, entre otros. Lo importante es la sincronización de las actividades en cuanto a calidad, costo y oportunidad de las diversas intervenciones.

Para que un ecosistema funcione productivamente, tiene que desarrollarse una cultura de trabajo colaborativo. Este elemento es probablemente el más complejo de lograr, pues tiene como gran componente la cultura de los diversos agentes, sus procesos y un componente imprescindible que hace que esa cultura pueda fluir; esa es la tecnología.

Es importante notar que el rol del Estado y los sistemas legislativos deben también redefinirse con el fin de facilitar el desarrollo de los ecosistemas y fomentar el desarrollo colaborativo; el Estado es un importante agente que debe participar activamente como facilitador.

Cada agente o elemento del ecosistema empresarial aporta una habilidad o conocimiento especializados con un valor absoluto y limitado, cuando el conjunto trabaja sincronizadamente, compartiendo información, procesos interempresariales y conocimiento, es el momento virtuoso de la generación de un valor ilimitado.

Este es pues el gran reto en el que se encuentran las empresas e instituciones líderes en el mundo y es lo que ocupa la energía de los inversionistas y directivos hoy y en los próximos años.

Comentamos que cada agente del ecosistema aporta una especialización o una característica única; entonces, uno de los primeros pasos es reconocer quiénes deben formar parte y qué aportes específicos se requieren. Para ello la empresa debe haber analizado detalladamente cada uno de sus procesos empresariales y unidades de negocio y definido cuáles son los esenciales, los que son una fortaleza no solo hoy sino mañana, que no se pueden imitar fácilmente y que forman parte de la real esencia de la empresa.

En este proceso de análisis, encontrará que muchas funciones pueden ser realizadas por otras empresas especializadas y que al tercerizar (outsource) un proceso o subproceso lograremos eficiencias en funciones y costos. Es así como hoy muchas empresas están en procesos agresivos de tercerización a empresas especializadas en finanzas y contabilidad, recursos humanos, tecnología de la información, telecomunicaciones, compras, logística, servicio a clientes, mantenimiento y servicios, etcétera. Esto proporciona flexibilidad, reducción de costos, capacidad de respuesta al mercado, siempre y cuando el trabajo sea colaborativo y funcione el ecosistema. 
Algo que puede graficar el modelo es una empresa cuya organización interna se va armando como piezas de "lego" de las partes que se contratan a terceros, pero quien da forma al proyecto y sabe ensamblar las partes es el arquitecto o director, creando y sumando algunas piezas claves y únicas.

La empresa puede inclusive cambiar de giro, abrir o cerrar líneas enteras de producción conservando lo que sabe hacer, su competencia esencial (core competency) sabe manejar empresas rentables, sabe cómo combinar todos los elementos y redireccionar la inversión de capital para que el negocio crezca, no para cumplir funciones que no le agregan valor.

El cambio cultural, de confianza, de conocimiento entre los agentes del ecosistema y el compartir procesos construyendo sobre estas experiencias de tercerización y sobre las alianzas estratégicas, va desarrollando el ambiente colaborativo, y se van viendo las ventajas. Por ejemplo, al desarrollar un nuevo servicio trabajando estrechamente con el cliente se lograrán mejores resultados en tiempo y costos, que si trabajamos independientemente, y a su vez nuestros proveedores, y presentamos reiteradas veces la propuesta al cliente. Este modo de trabajo conjunto es el concepto que reemplaza y enriquece aquel de "lealtad del cliente" al que muchos extrañan.

\section{Colaboración e innovación}

Colaboración es trabajar abiertamente desde un principio, todos con un objetivo común y respetando los intereses de cada quien.

La innovación y la colaboración no crecen donde la rutina abunda. Algunas preguntas que debemos hacernos para no caer en esa rutina son:

- ¿Me siento cómodo con la empresa y con la forma como la manejo?

- ¿Soy reconocido como un innovador?

- ¿Soy un ejemplo para la gente de la empresa?

- ¿Qué cambios innovadores estamos haciendo, qué nuevos productos y servicios?, ¿son simplemente mejoras o son cambios radicales?

- ¿Hacemos cambios en procesos operativos?

- ¿Cuántas nuevas alianzas y formas de interactuar con los miembros del ecosistema empresarial hemos desarrollado?

- ¿Qué rol juego en el ecosistema?, ¿cómo influyo? 
- ¿Qué tanto conocemos de tecnología y cómo la aplicamos?

- ¿Sacamos realmente ventaja de las inversiones realizadas en tecnología?, ¿cuáles?

- ¿Estamos trabajando de forma colaborativa?, ¿cómo desarrollamos estas relaciones?, ¿cómo estructuramos los aprendizajes y conocimiento de la empresa?, ¿cómo lo valorizamos?

La gran aliada en este punto es la tecnología, cuya evolución muy cercana al campo empresarial da encuentro a los nuevos modelos de negocio. Y son tres elementos que hacen posible todo este cambio: las telecomunicaciones, la infraestructura tecnológica de información y los estándares abiertos.

Es interesante destacar que el desarrollo exponencial en estas áreas se dio porque fueron las empresas de tecnología las primeras en incursionar en el campo colaborativo. Con el gran objetivo de desarrollar nuevos y mayores mercados, pusieron a disposición de sus competidores y del mercado en general, los estándares técnicos que hasta antes habían sido tratados como secretos de Estado, y esto lo hicieron con la finalidad de que todos desarrollen interfaces y nuevos elementos para que las distintas plataformas sean "interconectables", donde antes eran islas imposibles de conectar.

\section{Tecnología e Internet}

Hoy toda empresa invierte en tecnología, es importante para subsistir e imprescindible para ser competitiva. Pero más importante que los porcentajes de inversión es su calidad. Es cómo el modelo tecnológico sirve de vía de comunicación para hacer que el modelo de negocio se virtualice y los procesos e información fluyan.

El ejecutivo de hoy debe estar al tanto de la tecnología y probarla, así no tenga seguridad de cuál será la siguiente en implantarse en su empresa, y es aún más importante que sepa cómo tener acceso a ella. Qué alternativas de contratación tiene para que no distraiga aquellos capitales que deben estar destinados a nuevas inversiones. Debe ir borrando conceptos como "se 'cayó' el computador", pues pronto será una frase tan lejana como lo es "se rayó el disco" para los jóvenes de hoy, y esto no porque dejen de darse fallas técnicas, sino porque hay tantas alternativas de trabajar sin interrupciones que resulta absurdo mantener los antiguos esquemas. 
Ya se están rompiendo las barreras de minicomputación y súpercomputación; hoy simplemente hay acceso y la capacidad necesaria. El ejecutivo de hoy no debe preocuparse por las capacidades que tiene instaladas sino por los accesos y flexibilidades con que cuenta.

Muchos elementos tecnológicos que hasta antes considerábamos privilegio de científicos y laboratorios o solo para las más grandes corporaciones, están hoy disponibles para la empresa y observamos cómo los utilizamos cada vez con mayor facilidad y cómo medimos su productividad. Entre estos elementos se cuentan:

- Los microprocesadores, con multiplicada potencia y cada vez más reducido tamaño. Según el Reporte Anual 2005 de Semiconductor Industry Association, el mundo produjo más microprocesadores que granos de arroz ese año, y a menores costos. Son prácticamente computadores microscópicos cuyo uso aun más intensivo en algunos campos, como la medicina, la identificación y otros, estamos próximos a ver.

- Dispositivos de almacenamiento de información, con tremendas capacidades. Según Nick Bontis y Jac Fitz-enz en el artículo "Intellectual Capital ROI: A Causal Map of Human Capital Antecedents and Consequents" publicado en el Journal of Intellectual Capital [1] las bases de datos del mundo se duplicarán cada 11 horas en el año 2010. Y, en paralelo, van apareciendo dispositivos cada vez más pequeños y de mayor capacidad para transportar la información que requerimos.

- Etiquetas RFID (tecnología de identificación por radiofrecuencia), cuyo uso recién comenzó a darse en volúmenes considerables y que simplifican los procesos de distribución y control de mercadería, hoy se han comenzado a usar en grandes volúmenes, con costos mucho más accesibles.

- Los equipos inteligentes, teléfonos con capacidades de procesamiento, tarjetas inteligentes, blackberries, palms, que según IDC existirán en el mundo 22.000 millones en el 2008. Aquí están pendientes desarrollos para adaptarse aún más a los requerimientos y simplicidad que busca el usuario.

- Las comunicaciones e Internet, que facilitan el trabajo a nivel mundial y que prácticamente han establecido un único horario, el de $24 \times 7$, incorporando culturas, lenguajes, economías y sistemas de los más diversos hacia un objetivo común, hacer negocios y generar conocimiento. En comunicaciones vemos cada vez mayores capacidades e integración de elementos en un solo canal (voz y datos, por ejemplo). 
Internet está en plena evolución y así como la utilizamos para procesos tan sencillos como comunicarnos por correo electrónico y enviar mensajes en tiempo real, también le damos usos más elaborados de información, tanto para poner a disposición conocimiento como para acceder a este. Hoy el 17 por ciento de la población mundial está de alguna forma conectada a internet.

\section{Impacto social}

Y contra lo que algunos creen en el sentido de que es solo para uso urbano y empresarial, encontramos casos, en todo el mundo, de microempresarios que se benefician de internet. Un caso especialmente interesante que conozco es el de un campesino de un poblado del Cusco, que antes de llevar su cosecha para la venta en la feria dominical, hace escala en una cabina de Internet para revisar los precios actualizados de sus productos.

Internet está generando una revolución social y económica, la revolución del conocimiento colaborativo, la disponibilidad y el acceso al conocimiento que antes era aún más privilegiado, la construcción del conocimiento por parte de personas de las más diversas realidades, que tal vez podríamos comparar con las primeras hazañas de intercambio en la antigüedad, que abrieron nuevos horizontes comerciales y culturales. Hoy son muchas las culturas que se interrelacionan y, sobre todo, que actúan todas ial mismo tiempo! La velocidad del cambio y el impacto son comprensibles.

Imaginemos la potencia de todas aquellas comunidades de gente que se "reúne" en Internet para compartir un tema de interés, una investigación, la construcción de una nueva teoría o el desarrollo de un nuevo producto entre expertos de múltiples empresas alrededor del mundo. Esto es lo que se está creando hoy; aún no está del todo maduro pero podemos estar seguros de que traerá insospechados beneficios.

Algo similar sucedió en la industria del software con la construcción del sistema operativo Linux. Se desarrollo colaborativamente y se accede a algunas versiones en forma gratuita. Su utilización se esta generalizando y alrededor de él se van creando nuevos servicios. Se transformó así toda la industria y se dieron más opciones a los usuarios.

Cuando pensamos en los cambios sociales que se unen a la evolución empresarial y tecnológica, la reflexión puede ser aún más retadora, con un tercio de la población que se integra aceleradamente al sistema 
económico global, con un proceso ambicioso y sistematizado de capacitación y desarrollo de talento. Hoy tenemos alrededor de 800 mil nuevos ingenieros cada año sólo en China e India, a quienes se suman millones de personas altamente capacitadas, concentración de centros de investigación y desarrollo, concentración de funciones de las grandes corporaciones, desarrollo de grandes empresas superespecializadas, que, con los volúmenes de negocios que manejan, sin duda seguirá creciendo, y pueden ser las preferidas en los procesos de tercerización.

Por otro lado, la población de algunos países se va haciendo mayor y requiere construir capas sociales y económicas más jóvenes, lo cual va generando corrientes migratorias importantes.

Todo esto desarrolla talento e inteligencia con mayor concentración en los países anfitriones y reta a países más atrasados hacia el desarrollo de una ventaja, un conocimiento, un producto o servicios únicos, a una especialización que se base no en la extracción de los recursos naturales sino en el valor agregado que sobre estos recursos desarrollen sus pobladores y que, al mismo tiempo, sirvan para generar conocimiento especializado.

Y no se puede dejar de pensar en el tremendo impacto social que tiene el uso de la tecnología en el campo de la medicina, no solo porque se han reducido las estadísticas de mortalidad, sino también porque se ha mejorado la calidad de vida y se ha transformado el concepto de salud, que ahora mira hacia la medicina preventiva.

En nuestra vida diaria hemos ido incorporando tecnología incluso con mayor fluidez. Esto, en las próximas generaciones, será aun más natural, por la facilidad en el uso de los dispositivos y por la actitud que busca descubrir y conocer, y enfrentar las situaciones con menos temor a fallar, tratando innumerables veces de conseguir la forma exitosa de lograr el objetivo, porque saben que tienen que insistir una y otra vez para avanzar. Son generaciones que se esfuerzan por su desarrollo intelectual y manejan un sentido práctico de las cosas.

En la educación también vemos grandes cambios en la tecnología disponible; sin embargo, aún no está incorporada del todo en las metodologías de enseñanza porque en la mayoría de escuelas aún se "enseña conocimiento" y no se "enseña a aprender", y es aquí donde se produce un quiebre entre la mentalidad de los niños y los jóvenes, que han desarrollado estilos de aprendizaje interactivos, tratando y descubriendo, haciendo uso de las más diversas herramientas que atraen su atención y con las cuales es muy difícil que el maestro tradicional pueda competir. 
En cuanto a capacitación profesional y técnica se han dado saltos significativos, tanto en capacitación interna de las empresas como a través de cursos, herramientas complementarias y programas completos de posgrado y especialización de parte de las universidades, y todo esto basado en herramientas colaborativas. Es así como alumnos dispersos en distintas ciudades, que tal vez se encuentren un par de veces al año, establecen relaciones y métodos de trabajo de equipo de la más alta eficiencia. Estas experiencias nos abren todo un horizonte positivo para dar un salto cualitativo en capacitación.

\section{Reflexiones finales}

Hoy conocemos las tendencias y sabemos que el futuro es retador por lo imprevisible, que la velocidad con que se despliega el conocimiento y la información es la gran diferencia de nuestra época, derribando fronteras y superando idiomas y culturas. Hoy estamos más capacitados para construir un mundo equitativo, con mejores niveles de vida, como nunca antes se vieron, donde el ser humano es el factor clave. Es un momento fascinante.

Nos quedamos entonces con el tremendo reto de incluir a la población que aún no tiene acceso masivo al conocimiento y con la certeza de que con tecnología podemos acelerar su incorporación.

Es difícil imaginar un país viable en las próximas décadas, si no desarrolla talento y conocimiento en su población, y son la tecnología y la empresa aliados imprescindibles en el esfuerzo. 
[1] Bontis, N. y Fitz-enz, J. (2002). Intellectual Capital ROI: A Causal Map of Human Capital Antecedents and Consequents. Journal of Intellectual Capital, 3 (3), pp. 223-247.

[2] Kennedy, Paul M. Preparing for the Twenty-first Century. Fontana Press, 1994.

[3] Naisbitt, John. Megatrends 2000. Reissue edition. Avon Books, enero de 1991.

[4] Friedman, Thomas. The World Is Flat: The Globalized World in the Twenty-first Century. Penguin Books Ltd., 6 de abril del 2006

[5] Steiner, George A. Strategic Planning. Free Presspapersbacks, 1997.

[6] IBM Corporation. "The Global CEO Study 2006" [en línea]. $<w w w . i b m . c o m / b s c / c e o s t u d y>$.

[7] Bas, Enric. Megatendencias para el siglo XXI: Un estudio Delfos. México: Fondo de Cultura Económica, 2004.

\section{Referencias web:}

Department of Trade and Industries (England). $<$ http://www.dti.gov.uk/innovation/>.

The Innovation Journal. The Public Sector Innovation Journal (Canada). $<$ http://www.innovation.cc/>.

Innovation Network. Building communities for innovation. (California, EE. UU.). <http://www.thinksmart.com/>.

Australian Government Department of Industry, Tourism and Resources, The National Innovation Website.

$<$ http://www.innovation.gov.au/>. 
Carmen Rosa Graham 